\title{
Comparison of two surgical methods for the treatment of optic pathway gliomas in the intraorbital segment: an analysis of long-term clinical follow-up, which evaluates the surgical outcomes
}

\author{
Peng Yang ${ }^{1}$, Hao-Cheng Liu ${ }^{1}$, E Qiu ${ }^{1}$, Wei Wang ${ }^{1}$, Jia-Liang Zhang ${ }^{1}$, Li-Bin Jiang ${ }^{2}$, Jun Kang ${ }^{1}$ \\ ${ }^{1}$ Department of Neurosurgery, Tongren Hospital of China Capital Medical University, Beijing, China; ${ }^{2}$ Department of Ophthalmology, Tongren \\ Hospital of China Capital Medical University, Beijing, China \\ Contributions: (I) Conception and design: P Yang; (II) Administrative support: P Yang, HC Liu; (III) Provision of study materials or patients: P Yang, \\ HC Liu; (IV) Collection and assembly of data: E Qiu, W Wang; (V) Data analysis and interpretation: JL Zhang, LB Jiang, J Kang; (VI) Manuscript \\ writing: All authors; (VII) Final approval of manuscript: All authors. \\ Correspondence to: Jun Kang, MD, PhD. Department of Neurosurgery, Tongren Hospital of China Capital Medical University, Chongwenmennei \\ Street, Dongcheng District, Beijing 100730, China. Email: junkang2015@163.com.
}

\begin{abstract}
Background: This study analyzes two kinds of surgical methods for the treatment of optic pathway gliomas (OPGs) in the intraorbital segment, as well as the surgical outcomes of OPGs.

Methods: The clinical data of 86 patients with OPGs treated in our center was analyzed retrospectively, and the clinical symptoms, treatment methods, and follow-up results were recorded.

Results: For OPGs in the intraorbital segment, the optic nerve sheath incision was performed to remove a tumor while retaining the optic nerve sheath in Group $1(n=36)$. The optic nerve sheath and the tumor were simultaneously removed without retaining the optic nerve sheath in group $2(n=50)$. The effects of the design of the surgical methods on the post-surgical outcome are: One patient (1/36, 2.8\%) had recurrence in Group 1 and one patient $(1 / 50,2.0 \%)$ had recurrence in group 2. The differences in the surgical outcomes of the two groups regarding exophthalmos, conjunctiva swelling, eye movement disorder, and ptosis were found to be statistically significant $(\mathrm{P}<0.05)$. Compared with group 2 , the surgical outcomes in the postoperative phase of Group 1 were more satisfactory.

Conclusions: The two surgical methods do not increase the recurrence rate of tumors. The resection of OPGs through an optic nerve sheath incision was found to be an ideal surgical method for reducing complications in the postoperative phase and for providing better surgical outcomes.
\end{abstract}

Keywords: Optic pathway gliomas (OPGs); optic nerve sheath; surgical methods; surgical outcomes

Submitted Dec 14, 2020. Accepted for publication Apr 16, 2021.

doi: $10.21037 /$ tp-20-451

View this article at: http://dx.doi.org/10.21037/tp-20-451

\section{Introduction}

Optic pathway gliomas (OPGs) are gliomas that originate in the visual pathway. Approximately $2 \%$ become intracranial gliomas, $3 \%$ to $5 \%$ become intracranial tumors in children and $1 \%$ become intraorbital tumors. Six percent of OPGs can invade any part of the optic nerve pathway-including the optic nerve, the optic chiasma, the optic tract, and the optic radiation - and seriously affect the quality of a child's life $(1,2)$. The treatment methods for OPGs mainly involve chemotherapy, radiotherapy and surgery. The difficulty in surgery is the method of removing the tumor while protecting the child's eye movement and avoiding surgical complications (3-7). OPGs tumors mostly grow inside the optic nerve sheath along the optic nerve. The 
forward growth of OPGs touches on the intraorbital structure, while the posterior growth touches on the optic canal and intracranial structures. Ophthalmologists usually resect tumors with the assistance of neurosurgeons. In order to remove tumors from the optic canal and intracranial segment, many centers use a method that involves making an incision in the optic nerve sheath and the dura mater (8). To remove OPGs from the orbital segment, an ophthalmologist will usually simultaneously remove the optic nerve sheath and the OPGs. Intraorbital tumors generally involve the structure of the common tendinous ring of the orbital apex. Excessive traction can result in postoperative eyeball movement disorder, bulbar conjunctival edema, and ptosis.

The growth mode of an OPG can be divided into three categories, based on its relationship with the optic nerve sheath: the expanded endogenous type, the expanded exogenous type and the invasive growth type. The purpose of this study is to evaluate an alternative surgical method for removing an intrathecal tumor only once the optic nerve sheath has been incised, and to compare the clinical applicability and surgical outcomes of this method with a surgical method that involves removing the optic nerve sheath and the optic nerve tumor simultaneously. We present the following article in accordance with the STROBE reporting checklist (available at http://dx.doi. org/10.21037/tp-20-451).

\section{Methods}

Eighty-six patients with OPGs who were admitted to the Department of Neurosurgery and Ophthalmology of Beijing Tongren Hospital of the Capital Medical University in China, from January 2003 to October 2017, were examined. The postoperative pathological diagnosis was consistent with OPGs. Among the 86 patients, 41 were male $(52.3 \%)$ and 46 were female $(47.7 \%)$. The median surgical age was six years (1-6 years), and the median follow-up time was 78.5 months (43.8-134.3 months).

A total of 36 patients-of whom 14 were males (38.9\%) and 22 were females $(61.1 \%)$ - that were diagnosed with intraorbital tumors and who received an optic nerve sheath incision were defined as Group 1. There were 36 cases in Group 1, including 25 cases of simple involvement in orbit and 11 cases of involvement in orbit, optic pathway and/or optic chiasm. The median age of surgery was five years (4-10 years); the median time of intraorbital surgery was 70.0 minutes (60.0-97.5 minutes); the median hospital stay was 19.0 days
(17.0-24.8 days) and the median follow-up time was 76.0 months (38.3-108.0 months).

A total of 50 patients-of whom 27 were males $(54.0 \%)$ and 23 were females (46.0\%) - that were diagnosed with intraorbital tumors and who received a non-incision optic nerve sheath (the nerve sheath was not cut) were defined as group 2. There were 50 cases in group 2, including 24 cases of simple involvement in orbit and 26 cases of involvement in orbit, optic pathway, and/or optic chiasm. The median age of surgery was seven years (3-18 years), the median time of intraorbital surgery was 105.0 minutes (95.0-142.5 minutes), the median hospital stay was 19.0 days (15.0-23.3 days), and the median follow-up time was 80.5 months (45.3-142.5 months).

The study was conducted in accordance with the Declaration of Helsinki (as revised in 2013). The study was approved by the ethics committee of Tongren Hospital of China Capital Medical University. The study sample included patients who were diagnosed with OPGs in our center and who required surgery. After having the operation steps explained to them, all patients gave their informed consent.

\section{Inclusion criteria}

(I) Patients diagnosed with intraorbital or cranio-orbital tumors through orbital MRI and CT examinations. The intraorbital tumor is defined as an expanded endogenous or exogenous type confined within the optic nerve sheath. (II) Postoperative pathological diagnosis of OPGs. (III) All patients underwent microsurgery at the Beijing Tongren Hospital of the Capital Medical University. (IV) Postoperative imaging follow-ups took at least six months, including MRI or CT imaging.

\section{Exclusion criteria}

(I) Patients diagnosed with serious diseases affecting other systems, who were unable to tolerate surgery or who refused surgical treatment, as well as patients with obvious residual vision, which should be conservatively observed or changed to other non-surgical treatments. (II) Intraorbital tumors invade the optic nerve sheaths and grow diffusely, so they do not allow for the optic nerve sheaths to be preserved. (III) Patients who received surgical treatment at another hospital before surgery in our hospital or who were transferred to another hospital for continued treatment after surgery in our hospital. (IV) Lack of follow-up data for more than six months after surgery. (V) 
Combined with other underlying diseases, patients whose life expectancy is less than six months.

\section{Data collection and evaluation}

The clinical, imaging, and follow-up data of patients who met the inclusion criteria was recorded and a summary analysis was made: (I) clinical data: gender, age, symptoms, course of disease, surgical methods, complications, etc. (II) Imaging data: orbital CT and MRI. (III) Follow-up data were reviewed during hospitalization and in outpatient clinics, specifically including improved conditions of clinical symptoms and imaging (CT or MRI).

\section{Treatment options}

All patients had cranio-orbital or intraorbital tumors and were diagnosed with OPGs after surgery. All intraorbital tumors were in the optic nerve sheath, belonged to the expanded endogenous or exogenous type and did not involve the optic nerve sheath. The trans fronto-orbital approach was used to surgically remove tumors from the orbital or cranio-orbital area. All patients were operated on by neurosurgeons and ophthalmologists, and the surgical team members all had more than ten years of surgical experience. For the OPGs located in the inner segment of the optic canal and intracranial subdural segment, surgery was performed by incising the optic nerve sheath and dura mater and then removing the OPGs.

For patients in Group 1 who had OPGs located in the intraorbital segment, the optic nerve sheath incision was performed, and the tumor was removed from the back of the eyeball to the common tendinous ring. All surgical procedures were performed in the optic nerve sheath, thereby reducing the external operation of the optic nerve sheath and pulling the extraocular muscles and nerves in the orbital apex area. For patients in Group 2 who had OPGs located in the intraorbital segment, optic nerve sheath non-incision (the nerve sheath was not cut) was performed, and the tumor and the optic nerve sheath were simultaneously removed from the back of the eyeball to the common tendinous ring, separating the intraorbital muscles, fatty nerves, and other structures along the outside of the optic nerve sheath.

\section{Statistical analysis}

The results of the normality tests, KolmogorovSmirnov and Shapiro-Wilks, reveal that all the variables did not follow a normal distribution. Therefore, nonparametric methods were used to analyze the data. In order to compare continuous variables between the groups, the Mann-Whitney test was conducted. The median and quartile [M (P25, P75)] were calculated from the measurement data of skewed distribution. Discrete variables were reported as proportions and percentages and compared by performing the Chi-square test or the Fisher's exact test. In order to compare the ratios between time points, the McNemar chi-square test was conducted. The SPSS Statistics 20.0 statistical software package was used for data processing. The statistical significance was set at a $\mathrm{P}$ value of less than 0.05 .

\section{Results}

\section{Clinical features}

Among the 86 patients, 45 (52.3\%) experienced simple exophthalmos symptoms, 14 (16.3\%) experienced simple vision loss or visual field defect symptoms, 27 cases experienced both symptoms of exophthalmos and of vision loss (31.4\%), and 5 cases (5.8\%) experienced hypothalamic symptoms (polydipsia, polyuria, and abnormal hormones).

\section{Imaging features of lesions}

The MRI imaging revealed that the tumor was located on the intraorbital segment in all 86 cases; in 64 cases it involved the optic canal, in 35 cases it involved the entire optic nerve and optic chiasm, and in one case it involved the posterior chiasm optic tract. The tumor appeared as a mass in the posterior muscular cone of the eyeball. The tumor was equal in signal to the brain parenchyma on the T1weighted image and in hyperintensity on the $\mathrm{T} 2$-weighted image. The tumor showed mild to moderate enhancement after the injection of contrast agents, and 20 cases showed uneven strengthening.

On the CT scan, all 86 cases showed equidensity with the brain parenchyma. Fifty cases had uniform density and a clear boundary, which was indistinguishable from the optic nerve. After enhancement, the tumor showed moderate improvement. The CT scan of 40 cases showed that the optic canal was enlarged.

\section{Treatment and follow-up results}

All 86 patients were treated with microsurgery, and all the 
Table 1 Patient characteristics and summary of clinical features of two groups

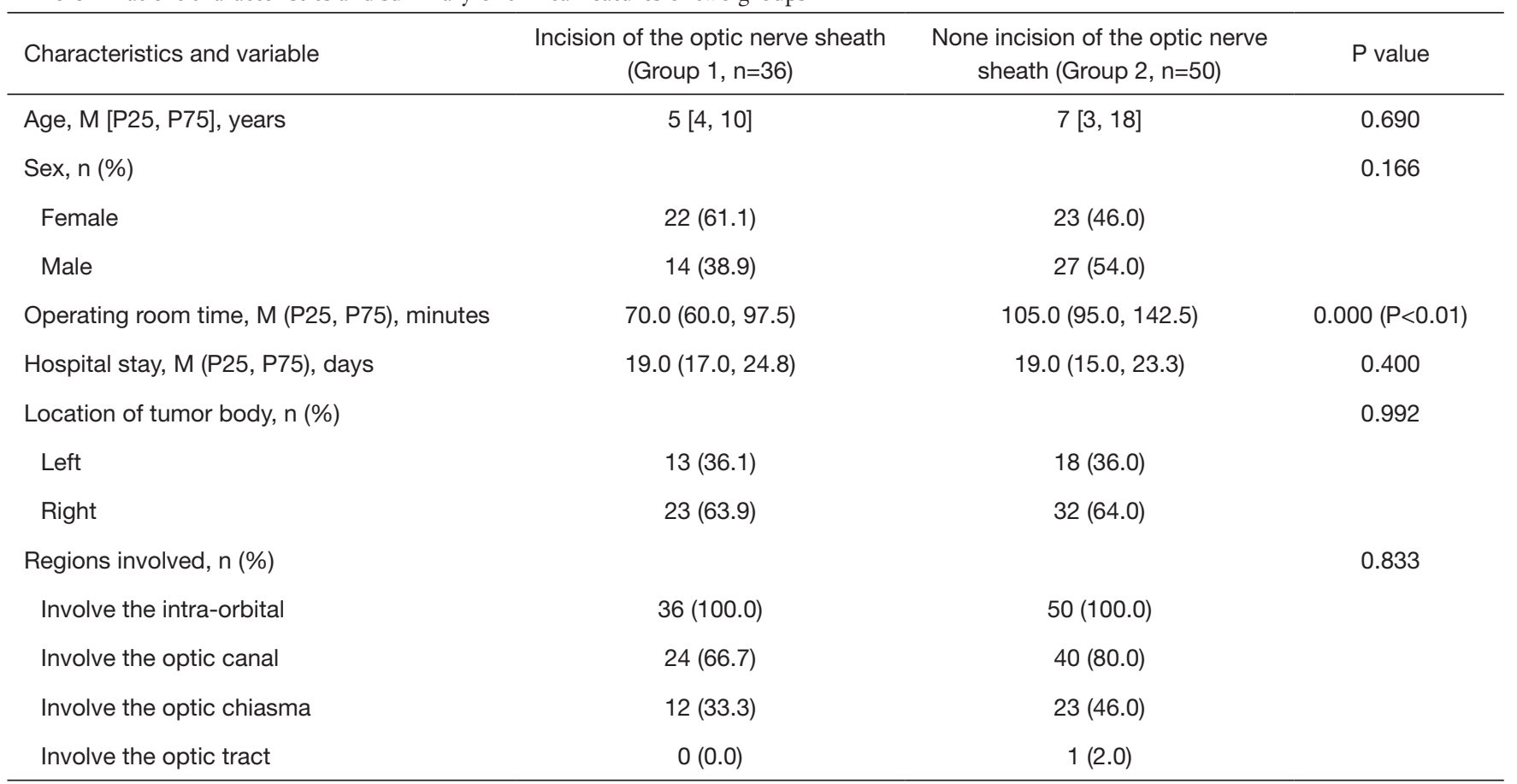

patients were treated using the trans fronto-orbital approach to remove intraorbital or cranio-orbital tumors. The follow-up results revealed that: two patients (2.3\%) (Group $1=1$; Group $2=1$ ) recurred, two patients $(2.3 \%)$ (Group 1 =1; Group $2=1$ ) experienced postoperative intracranial infection, one patient $(1.2 \%)$ (Group $2=1$ ) experienced contralateral visual field defect, 67 patients (77.9\%) (Group $1=27$; Group $2=40$ ) experienced ptosis, 73 patients $(84.9 \%)$ (Group 1 =28; Group $2=45$ ) experienced eyeball movement upward disorder, 24 patients (27.9\%) (Group 1 =5; Group $2=24$ ) experienced eyeball movement downward disorder, 24 patients $(27.9 \%)$ (Group $1=5$; Group $2=24$ ) experienced abduction disorder, 24 patients (27.9\%) (Group 1 =5; Group $2=24)$ experienced adduction disorder, and 25 patients (Group 1 =6; Group 2 =19) experienced conjunctival edema.

We followed up the preoperative and postoperative conditions of the two groups of patients, such as the ptosis, eyeball movement upward, eyeball movement downward, adduction movement, abduction movement, and the conjunctival edema caused by the two different surgical methods. We recorded symptoms on the first day, seventh day, thirtieth day and ninetieth day after surgery. We also followed up the tumor recurrence caused by the two different surgical methods over a prolonged period, and compared the images at three months, one year and three years after the operation.

Patients who received an incision in the optic nerve sheath and intrathecal resection of the tumor (Group 1) were compared with patients who did not undergo an incision of the optic nerve sheath to remove the tumor (Group 2). On the first and seventh day after surgery, there was no significant difference in the eyeball movement upward disorder and ptosis. However, the condition of Group 1 was better than that of Group 2 from day 7 to three months after the surgery, with a statistical difference of $(\mathrm{P}<0.05)$. The surgical outcomes—such as eyeball abduction, eyeball adduction, eyeball movement downward and conjunctival edema-of Group 1 were better than Group $2(\mathrm{P}<0.05)$. Furthermore, the different surgical methods of the two groups did not affect the prognosis of the operation and tumor recurrence $(\mathrm{P}>0.05)$ (Tables 1 and 2$)$.

\section{Typical case 1}

This case involves a one-year-old female who was admitted to our hospital due to progressive right eye proptosis over a period of six months. An orbital MRI revealed a right cranio-orbital communication optic nerve tumor, which involved the inner orbital segment, the inner segment of the optic canal and the intracranial segment of the visual pathway. The right eye was exophthalmos on admission and 
Table 2 Complications and recurrence of two groups

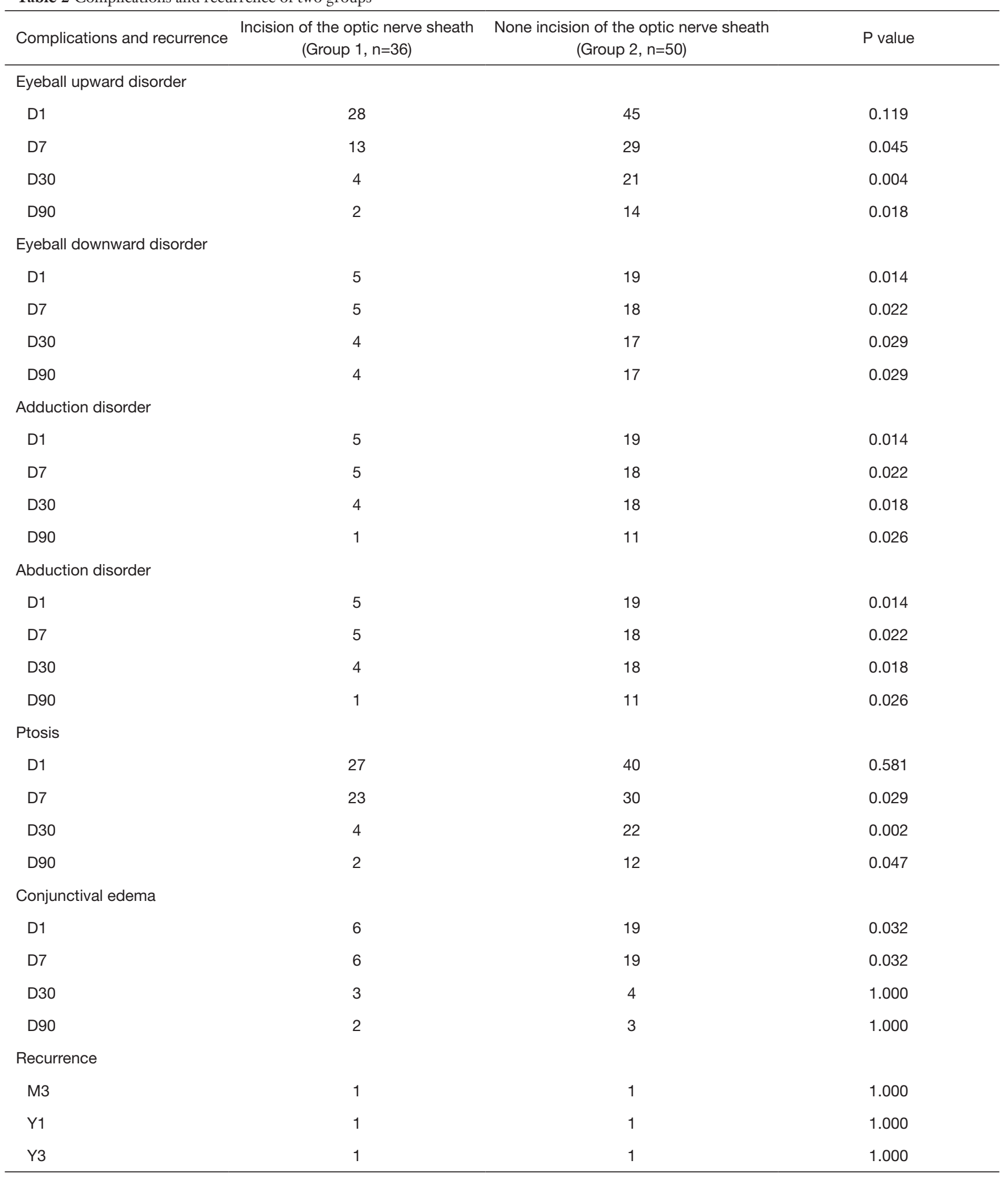


the right eyeball movement was normal in all directions. The right pupil was $5 \mathrm{~mm}$ in diameter and the right eye vision had no light perception; its direct light reaction had disappeared, while its indirect light reaction continued to function. The craniotomy of the trans fronto-orbital approach was adopted to remove the cranio-orbital communication tumours. Tumors located in the optic canal and intracranial segments were excised subdurally after an incision of the dura mater, and the tumors were removed from the subdural anteriorly to the common tendinous ring. During the operation, the ophthalmic artery was preserved in the optic nerve sheath. The intraorbital tumor was then removed through the optic nerve sheath incision from the back of the eyeball to the common tendinous ring. Finally, the whole orbital tumor was removed. On the first day after the operation, the patient only suffered from mild ptosis and eyeball movement upward disorder. All directions of the right eyeball movement were back to normal on the seventh, thirtieth and ninetieth day after the operation (Figure 1).

\section{Typical case 2}

This case involves a nine-year-old female who was admitted to our hospital due to progressive right eye proptosis with decreased vision for a period of one month. An orbital MRI revealed a right cranio-orbital communication optic nerve tumor, which involved the inner orbital segment and the inner segment of the optic canal. The right eye was exophthalmos on admission; however, the eyeball movement was normal in all directions. The right pupil was $5 \mathrm{~mm}$ in diameter, and the eye had no light perception only indirect light reaction remained. The craniotomy of the trans fronto-orbital approach was adopted to remove cranio-orbital communication tumors. Tumors in the optic canal were excised subdurally through the incision of the dura mater, and the tumors were removed from the subdural anteriorly to the common tendinous ring. The ophthalmic artery was preserved in the sheath. The intraorbital tumor was separated from the main body of the tumor with non-incision (the nerve sheath was not cut) of the optic nerve sheath. The intraorbital operation was performed outside the sheath, from the back of the eyeball to the common tendinous ring. On the first day after the operation, the patient suffered from right eye ptosis and right eyeball movement disorder in all directions. On the seventh and thirtieth day after the operation, there was no reduction in the eyeball movement disorder. The right eye ptosis improved on the ninetieth day, but the eyeball movement did not return to normal (Figure 2).

\section{Discussion}

Although OPGs are common in children, they can be found in patients of all ages (8). They are often difficult to diagnose at an early stage due to their insidious onset and diverse symptoms. They are often accompanied by severe ophthalmological or hypothalamic symptoms during treatment. The onset of these tumors is usually indicated by unilateral ocular symptoms, and exophthalmos and decreased vision are common clinically. It is relatively rare for the tumor to be in both eyes (9). There are still controversies about the timing and the effects of surgery. Previously, OPGs were often treated with surgery (10); however, with the in-depth study of OPGs and the rise of chemotherapy, surgical resection is no longer the mainstream method. Sawamura et al. (11) believe that it is almost impossible to cure tumors with simple surgical resection, and that they should be kept under control through chemotherapy and radiotherapy.

There is no reliable data that can be used to compare the efficacy of chemotherapy alone with that of surgery combined with chemotherapy (12). As there is no effective targeted therapy, chemotherapy can only be used as a supplement to surgery. Goodden et al. (13) believe that the indications for surgery should include: (I) progressive deterioration of vision and eye exophthalmos, (II) huge, cystic tumors that make it difficult to reduce the space-occupying effect through chemotherapy, (III) serious tumor blockage caused by ventricular system hydrocephalus, and (IV) damage to the hypothalamic-pituitary endocrine axis, especially in infants. However, in recent years, many centers have explored surgical treatment of OPGs, and have achieved significant surgical results for intractable OPGs $(14,15)$.

Therefore, it is necessary to summarize the clinical characteristics, treatment principles, and prognosis of OPGs in order to better understand them and the effects of treatment. OPGs are a type of low-grade astrocytoma (WHO grade I-II). According to related studies $(16,17)$, OPGs account for approximately one percent of all central nervous system tumors and three to five percent of brain tumors in children. At present, most scholars worldwide use the Dodge classification standard, which divides OPGs into three types based on their MRI: type I tumors, which only involve unilateral or bilateral optic nerves; type II tumors, which invade the optic chiasm, with or without involving the optic nerve proximity structure; and type 

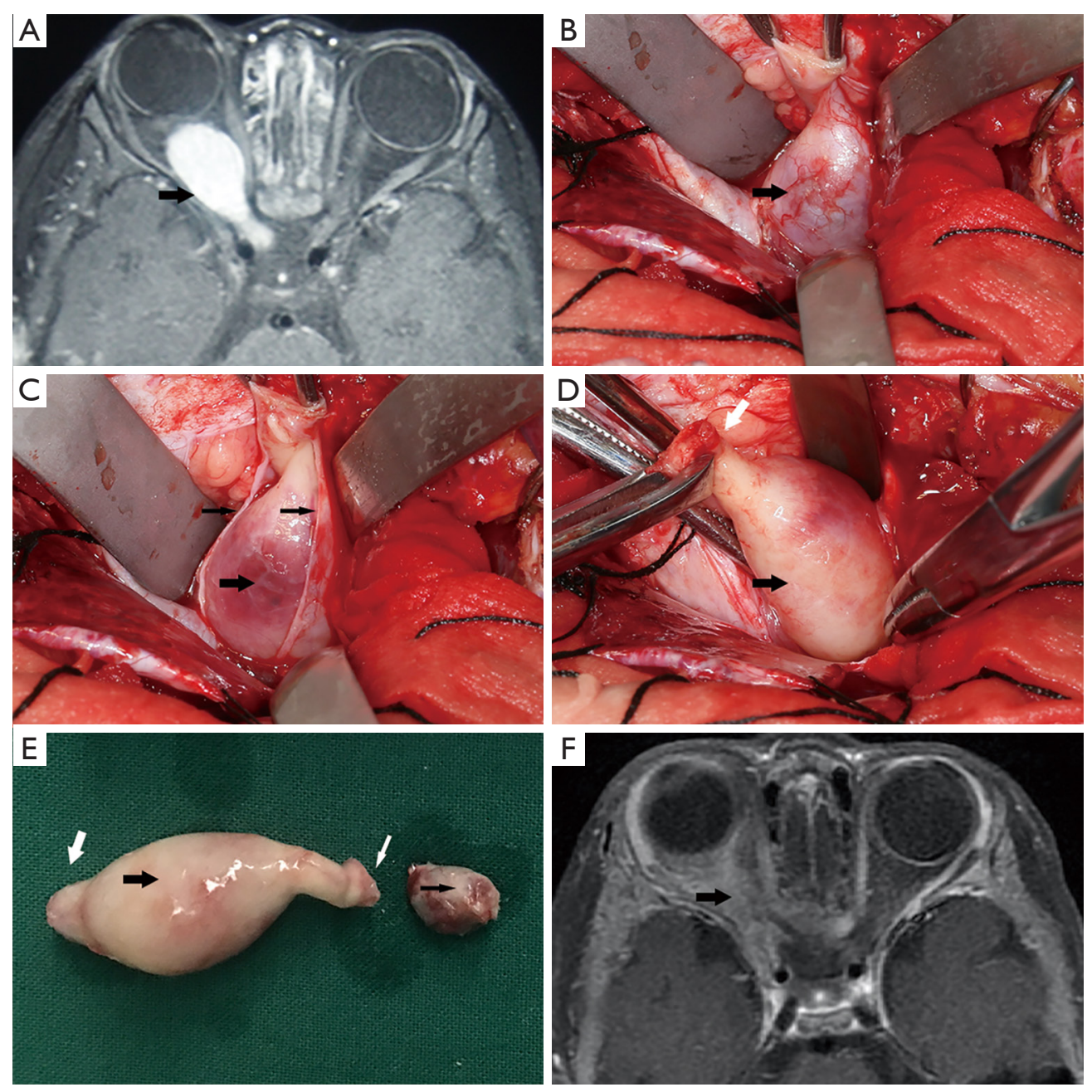

Figure 1 A 1-year-old female who was admitted to our hospital due to progressive right eye proptosis for 6 months. (A) Orbital MRI showed that the right cranio-orbital communication optic nerve tumor, the tumor involved the inner orbital segment, the inner segment of the optic canal, and the intracranial segment of the visual pathway (black thick arrow). (B) We gave a craniotomy of transfronto-orbital approach to remove the cranioorbital communication tumor, and exposed the tumor in the orbital segment outside the optic nerve sheath (black thick arrow). (C) The optic nerve sheath was incised (black thin arrow) to expose the tumor body (black thick arrow) in the sheath, from the back of the eyeball to the common tendinous ring. (D) We separated the main body of the tumor in the sheath, and removed the whole orbital tumor part (black thick arrow). (E) The intraorbital optic glioma tumor was completely resected (black thick arrow), and the optic nerve sheath was preserved. The two ends of the tumor were the posterior bulbar segment (white thin arrow) and the common tendinous ring segment (white thick arrow). The intracranial tumor was completely removed (black thin arrow). (F) Compared with (A), postoperative orbital enhanced MRI showed complete resection of the tumor (black thick arrow).

III tumors, which invade the hypothalamus and adjacent structures. For intracranial tumors, many centers have consistent opinions on which surgery should be performed, namely, the subdural resection of tumors (18). However, for intraorbital OPGs, most studies do not mention any specific surgical methods. Surgery can increase the risk of blindness, so surgery is generally only used as a treatment for advanced OPGs.

OPGs are more common in children, and many centers have conducted retrospective studies (19,20). Although chemotherapy had good results on patients with vision preservation, the patients in our center had already suffered from severe visual loss or even blindness when they came to the clinic. Ensuring that the tumor is completely removed 

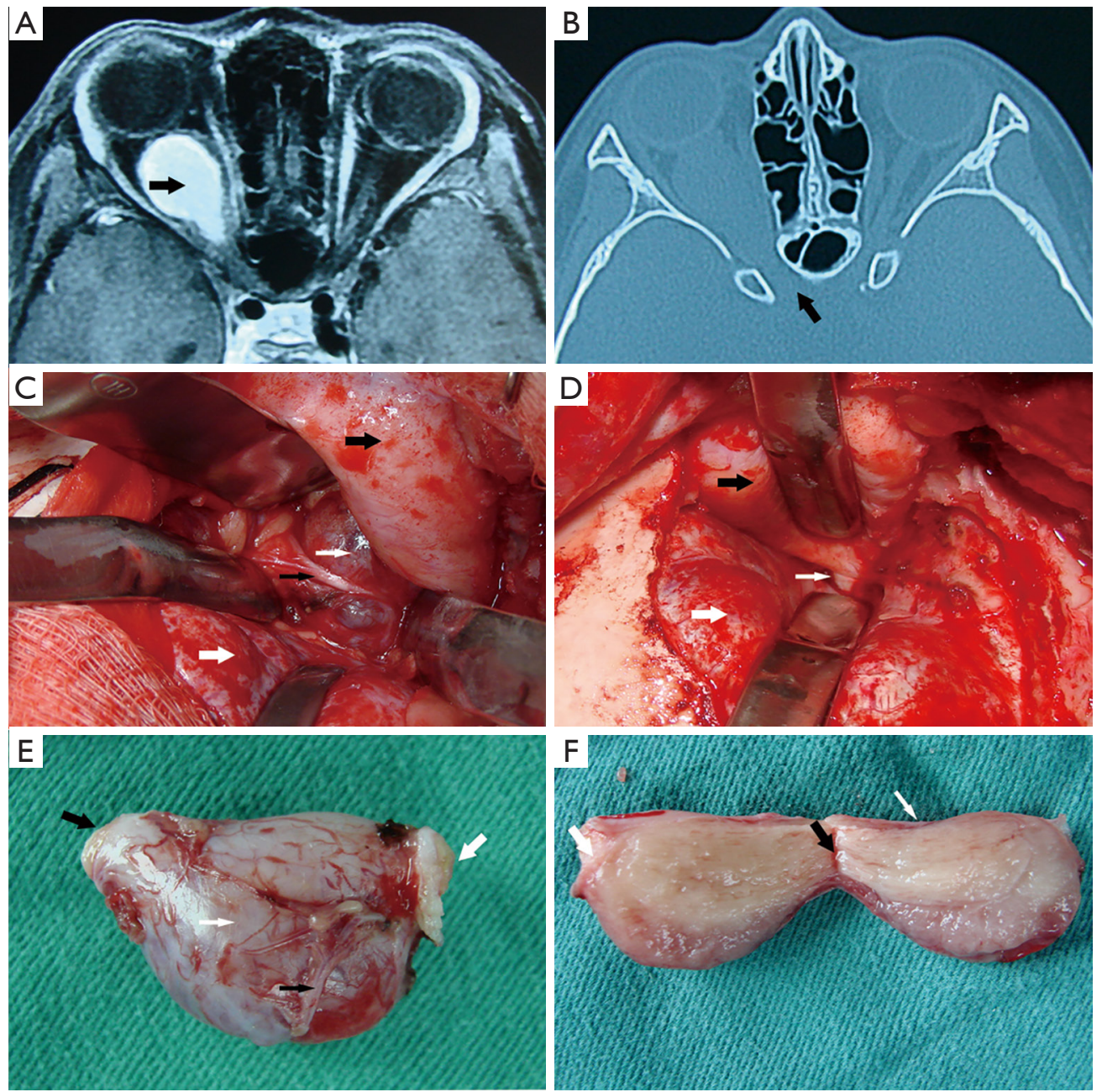

Figure 2 A 9-year-old female who admitted to our hospital due to progressive right eye proptosis with the decreased vision for 1 month. (A) Orbital MRI showed that the right cranioorbital communication optic nerve tumor, the tumor involved the inner orbital segment, the inner segment of the optic canal (black thick arrow). (B) Thin-layer CT scan of the optic canal showed that the right optic canal was enlarged than the left (black thick arrow). (C) We gave a craniotomy of transfronto-orbital approach to remove the cranioorbital communication tumor. The intra-orbital tumor was bluntly separated outside the sheath with the none incision (the nerve sheath was not cut) of the optic nerve sheath. The operation was performed between the orbital contents (black thick arrow) and the frontal epidural (white thick arrow), we could see that the first branch of the trigeminal nerve (black thin arrow) spanned the tumor body (white thin arrow). (D) During the operation, we could see a thickened optic canal tumor (white thin arrow) between the orbital contents (black thick arrow) and the frontal epidural (white thick arrow), which was consistent with preoperative CT of the optic canal. The optic nerve located in the optic canal was thickened (white thin arrow). (E) The tumor was removed together with the optic nerve sheath from the back of the eyeball (black thick arrow) to the common tendinous ring (white thick arrow). After the whole orbital tumor was removed, the first branch of the trigeminal nerve was also removed. Optic nerve sheath was not preserved (white thin arrow), and the branch of the V1 was not preserved (black thin arrow). (F) The resected tumor body was traversed between the back of eyeball (black thick arrow) and the common tendinous ring (white thick arrow), and the optic nerve sheath outside the tumor body (white thin arrow) could be seen.

and that the eyeball movement function and the appearance of the patient is preserved is especially important for children's quality of life. The optic nerve can be divided into four parts: intra-balloon, orbital, intracanal and intracranial. The outer optic nerve sheath has three layers of membranes that are continuous with the corresponding three layers 
of the meninges: the dura mater, pia mater and arachnoid membranes, respectively. The front end of the subdural and subarachnoid space is the blind end, and it ends behind the eyeball. The sheath space is the same as the brain space with the same name, and it is filled with cerebrospinal fluid. The blood supply to the optic nerve mainly comes from the ophthalmic artery. The intraorbital optic nerve is a continuation of the intracranial optic nerve and has the same anatomical basis.

Therefore, we argue that the surgical method of the intracranial segment must be suitable for the surgical method of intraorbital tumors. For ophthalmologists, the anatomy of the cranio-orbital area is particularly important; the exposure and anatomy of the common tendinous ring and orbital apex area have a great influence on the prognosis of the patient (21). Considering the method of craniotomy, which completely exposes the entire optic nerve of the cranio-orbital area, Natori, Rhoton and Shimizu et al. $(22,23)$ argues that there are three main surgical methods for exposing the structure of the orbital area of tumors in the cranio-orbital area, i.e., through the superior oblique muscle, the superior rectus muscle and the external rectus muscle. The space of the frontal nerve exposes the tumor to the deep part of the orbit, and the trans fronto-orbital craniotomy can access these paths. Currently, there are no clear guidelines for deciding which patients with OPGs require surgery, but the surgical patients selected by our center were mainly patients with severe exophthalmos that affected their appearance, blindness of the affected eye or vision less than 0.01 and involved the optic chiasm and distal optic tract structure.

In order to ensure the accuracy of the data on the postoperative complications of an intraorbital tumor, the operations we adopted for the intracanal and intracranial tumors involved unified subdural surgery to remove the tumor. The removal of the intracranial tumor involved cutting the dura mater and severing the optic nerve at the front of the optic chiasm or at the back of the tumor. The removal of the tendinous ring tumor located in the intracanal segment involved cutting the dorsal sheath of the optic canal to the boundary of the common tendinous ring, removing the tumor and then preserving the ophthalmic artery. The removal of an intraorbital tumor involved making an incision on the eyebrow arch and the frontotemporal bone flap, separating the orbital roof epidurally and removing the bones, the optic canal, and superior orbital fissure. The orbital periosteum was cut longitudinally on the midline of the orbital roof.
We used an operating microscope to cleanly separate the lateral space of the superior rectus muscle; we looked for the front end of the tumor, retracted the superior rectus muscle, and fully separated the tumor head. We performed two surgical methods: the first (Group 1) involved finding the tumor in the orbit, incising the optic nerve sheath, separating the tumor in the optic nerve sheath from the back of eyeball to the common tendinous ring, only removing the tumor from the optic nerve sheath and retaining the optic nerve sheath to reduce additional damage caused by separating the orbital apex structure outside the sheath. The second method (Group 2) involved finding the tumor in the orbit. To do this, we separated the tumor through the outer side of the optic nerve sheath from the back of the eyeball to the common tendinous ring. This method of blunt separation was likely to cause postoperative eyeball movement disorders and ptosis, and multiple traction could cause serious postoperative bulbar conjunctival edema. In the follow-up of the eyeball movement disorder and appearance of the two groups of patients, there was no difference in the incidence of ptosis and eyeball upward movement disorder on the first day after the operation. On the seventh, thirtieth and ninetieth days after the operation, the ptosis and eyeball upward movement disorder of Group 1 had improved more than that of Group 2.

Regarding the function of adduction, abduction, downward movement and bulbar conjunctival edema, Group 1 improved more than Group 2 at all the time points during the follow-up. We believe that the two surgical methods should use the inner and outer space of the superior rectus muscle to expose the tumor in the orbit. Neither surgical method can avoid short-term surgical traction that causes transient postoperative eyeball upward movement disorder and ptosis. Therefore, both surgical methods will have a certain impact on the eyeball upward movement disorder and ptosis in the short term after the operation. However, in the long term, in Group 1, the degree of traction was light, there was almost no damage to the orbital apex structure, and the long-term effect of postoperative eyeball movement disorder was improved. The common tendinous ring runs through the extraocular muscles, optic nerve, oculomotor nerve, trochlear nerve, abductor nerve and other structures. The surgical method adopted in Group 2 involves exposing the orbital tumor out of the sheath, which leads to an increase in the time of surgical separation, the scope of exposure, and possible collateral injuries. An incision on the common tendinous ring structure increases the risk of postoperative eyeball 
movement disorder. In this study, all tumors were primarily located in the sheath, and the cumulative sheath and extrathecal structure of the tumor were not discussed in surgical terms because whether the optic nerve sheath was retained depended on the intraoperative analysis.

In general, the entire body of the tumor showed colloidal changes during the operation. Some tumors were accompanied by cystic changes, while some tumors showed intratumoral hemorrhage. Such changes have also been reported in other studies $(24,25)$. As a low-grade glioma, the overall prognosis of OPGs was good, and the five-year survival rates reported by different centers were all above $70 \%$. In this study, only two cases of tumors progressed, and the postoperative pathology was glioblastoma (WHO IV) with spinal cord dissemination. As for the factors affecting the prognosis of children with OPGs, there is currently no unified conclusion. Many scholars believe that whether to undergo postoperative radiotherapy is an independent factor affecting the progression-free survival of children. Other studies believe that age plays a significant role in the prognosis of children (26); the prognosis of children less than three years old was significantly worse (7).

In recent years, many articles on the natural history of children's OPGs have been released (27). These studies confirm the complexity of OPGs. NF1 is found to be the main determinant of tumor behavior. However, most studies promote conservative treatment, radiotherapy and chemotherapy follow-up, and none include information on OPG surgery. There are also some cutting-edge studies which analyze the incidence of OPGs and ethnicity and argue that white people are the most affected (28). Liu et al. (29) also point out the importance of multidisciplinary cooperation in the treatment of OPGs.

In this study, which includes follow-up observations from three to seventeen years of postoperative patients, only two cases experienced tumor recurrence. The two surgical methods had no statistical difference in the tumor recurrence rate of patients, and both surgical methods could give a complete resection of the tumor. The overall recurrence rate of the operation was $2.3 \%$, so the surgical method of Group 1 could effectively improve the patient's quality of life without increasing the tumor recurrence rate. Since most children with OPGs can survive for a long time, preserving or improving their quality of life should be a priority during treatment. Important factors that affect children's quality of life include visual acuity, eyeball movement function and endocrine function. In this study, by changing the traditional surgical methods for intraorbital tumors could significantly improve patients' quality of life after surgery.

The surgical indications of OPGs must be kept strictly under control, and multidisciplinary cooperation between ophthalmology and neurosurgery is required to evaluate the overall condition of the patient. The overall surgical prognosis of OPGs is good, and most patients can survive for a long time. It is particularly important to ensure the quality of life of patients, especially that of children, by ensuring normal eyeball movement function and preserving their appearance after an operation. Both surgical methods can completely remove a tumor and do not increase the recurrence rate. Intraorbital tumor resection with optic nerve sheath incision is found to be an ideal surgical method for reducing postoperative complications and achieving better surgical outcomes.

\section{Acknowledgments}

This manuscript has been proofread and edited by a professional English editing company from Proofed Inc. Funding: None.

\section{Footnote}

Reporting Checklist: The authors have completed the STROBE reporting checklist. Available at http://dx.doi. org/10.21037/tp-20-451

Data Sharing Statement: Available at http://dx.doi. org/10.21037/tp-20-451

Peer Review File: Available at http://dx.doi.org/10.21037/tp$20-451$

Conflicts of Interest: All authors have completed the ICMJE uniform disclosure form (available at http://dx.doi. org/10.21037/tp-20-451). The authors have no conflicts of interest to declare.

Ethical Statement: The authors are accountable for all aspects of the work in ensuring that questions related to the accuracy or integrity of any part of the work are appropriately investigated and resolved. The study was conducted in accordance with the Declaration of Helsinki (as revised in 2013). The study was approved by ethics committee of Tongren Hospital of China Capital Medical University. After explaining the operation steps, all patients 
gave their informed consent.

Open Access Statement: This is an Open Access article distributed in accordance with the Creative Commons Attribution-NonCommercial-NoDerivs 4.0 International License (CC BY-NC-ND 4.0), which permits the noncommercial replication and distribution of the article with the strict proviso that no changes or edits are made and the original work is properly cited (including links to both the formal publication through the relevant DOI and the license). See: https://creativecommons.org/licenses/by-nc-nd/4.0/.

\section{References}

1. Janss AJ, Grundy R, Cnaan A, et al. Optic pathway and hypothalamic/chiasmatic gliomas in children younger than age 5 years with a 6 -year follow-up. Cancer 1995;75:1051-9.

2. Mandiwanza T, Kaliaperuma A, Sattar M, et al. Suprasellar pilocytic astrocytoma: one national centre's experience. Childs Nerv Syst 2014;30:1243-8.

3. Thomas RP, Gibbs IC, Xu LW, et al. Treatment options for optic pathway gliomas. Curr Treat Options Neurol 2015;17:333.

4. Acharya S, Quesada S, Coca K, et al. Long-term visual acuity outcomes after radiation therapy for sporadic optic pathway glioma. J Neurooncol 2019;144:603-10.

5. Dodgshun AJ, Elder JE, Hansford JR, et al. Long-term visual outcome after chemotherapy for optic pathway glioma in children: Site and age are strongly predictive. Cancer 2015;121:4190-6.

6. Silva MM, Goldman S, Keating G, et al. Optic pathway hypothalamic gliomas in children under three years of age: the role of chemotherapy. Pediatr Neurosurg 2000;33:151-8.

7. Tsang DS, Murphy ES, Merchant TE. Radiation Therapy for Optic Pathway and Hypothalamic Low-Grade Gliomas in Children. Int J Radiat Oncol Biol Phys 2017;99:642-51.

8. Heiland T, Karpel-Massler G, Scheuerle A, et al. Rare Case of Sporadic Malignant Optic Pathway Glioma in 71-Year-Old Woman. World Neurosurg 2020;133:413-5.

9. Karaaslan B, Uçar M, Kulduk G, et al. Bilateral Optic Pathway Ganglioglioma: The Fifth Case in the Literature. Pediatric Neurosurgery 2016;51:48-54.

10. Konovalov A, Gorelyshev S, Serova N. Surgery of giant gliomas of chiasma and IIIrd ventricle. Acta Neurochir (Wien) 1994;130:71-9.

11. Sawamura Y, Kamada K, Kamoshima Y, et al. Role of surgery for optic pathway/hypothalamic astrocytomas in children. Neuro Oncol 2008;10:725-33.

12. Jahraus CD, Tarbell NJ. Optic pathway gliomas. Pediatr Blood Cancer 2006;46:586-96.

13. Goodden J, Pizer B, Pettorini B, et al. The role of surgery in optic pathway/hypothalamic gliomas in children. J Neurosurg Pediatr 2014;13:1-12.

14. Hidalgo ET, Kvint S, Orillac C, et al. Long-term clinical and visual outcomes after surgical resection of pediatric pilocytic/pilomyxoid optic pathway gliomas. J Neurosurg Pediatr 2019;24:166-73.

15. Liu Y, Hao X, Liu W, et al. Analysis of Survival Prognosis for Children with Symptomatic Optic Pathway Gliomas Who Received Surgery. World Neurosurg 2018;109:e1-e15.

16. Trevisson E, Cassina M, Opocher E, et al. Natural history of optic pathway gliomas in a cohort of unselected patients affected by Neurofibromatosis 1 . J Neurooncol 2017;134:279-87.

17. Listernick R, Ferner RE, Liu GT, et al. Optic pathway gliomas in neurofibromatosis-1: controversies and recommendations. Ann Neurol 2007;61:189-98.

18. Borghei-Razavi H, Shibao S, Schick U. Prechiasmatic transection of the optic nerve in optic nerve glioma: technical description and surgical outcome. Neurosurg Rev 2017;40:135-41.

19. Doganis D, Pourtsidis A, Tsakiris K, et al. pathway glioma in children: 10 years of experience in a single institution. Pediatr Hematol Oncol 2016;33:102-8.

20. El Beltagy MA, Reda M, Enayet A, et al. Treatment and Outcome in 65 Children with Optic Pathway Gliomas. World Neurosurg 2016;89:525-34.

21. Martins C, Costa E Silva IE, Campero A, et al. Microsurgical anatomy of the orbit: the rule of seven. Anat Res Int 2011;2011:468727.

22. Natori Y, Rhoton ALJ. Transcranial approach to the orbit: microsurgical anatomy. J Neurosurg 1994;81:78-86.

23. Shimizu S, Tanriover N, Rhoton AL, et al. MacCarty keyhole and inferior orbital fissure in orbitozygomatic craniotomy. Neurosurgery 2005;57:152-9.

24. Serova NK, Konovalov AN, Eliava SS, et al. Chiasm and optic nerve glioma manifested as hemorrhage (two clinical cases and a literature review). Zh Vopr Neirokhir Im N N Burdenko 2016;80:90-7.

25. van Baarsen K, Roth J, Serova N, et al. Optic pathwayhypothalamic glioma hemorrhage: a series of 9 patients and review of the literature. J Neurosurg 2018;129:1407-15. 
26. Walker DA, Liu J, Kieran M. A multi-disciplinary consensus statement concerning surgical approaches to low-grade, high-grade astrocytomas and diffuse intrinsic pontine gliomas in childhood (CPN Paris 2011) using the Delphi method. Neuro Oncol 2013;15:462-8.

27. Nicolin G, Parkin P, Mabbott D, et al. Natural history and outcome of optic pathway gliomas in children. Pediatr Blood Cancer 2009;53:1231-7.

28. Peckham-Gregory EC, Montenegro RE, Stevenson

Cite this article as: Yang P, Liu HC, Qiu E, Wang W, Zhang JL, Jiang LB, Kang J. Comparison of two surgical methods for the treatment of optic pathway gliomas in the intraorbital segment: an analysis of long-term clinical followup, which evaluates the surgical outcomes. Transl Pediatr 2021;10(6):1586-1597. doi: 10.21037/tp-20-451
DA. Evaluation of racial disparities in pediatric optic pathway glioma incidence: Results from the Surveillance, Epidemiology, and End Results Program, 2000-2014. Cancer Epidemiol 2018;54:90-4.

29. Liu APY, Hastings C, Wu S, et al. Treatment burden and long-term health deficits of patients with low-grade gliomas or glioneuronal tumors diagnosed during the first year of life. Cancer 2019;125:1163-75. 